\title{
Comparison of the quasi-static method and the dynamic method for simulating fracture processes in concrete
}

\author{
J. X. Liu - S. C. Deng · N. G. Liang
}

Received: 15 January 2007 / Accepted: 24 September 2007 / Published online: 27 October 2007

(C) Springer-Verlag 2007

\begin{abstract}
Concrete is heterogeneous and usually described as a three-phase material, where matrix, aggregate and interface are distinguished. To take this heterogeneity into consideration, the Generalized Beam (GB) lattice model is adopted. The GB lattice model is much more computationally efficient than the beam lattice model. Numerical procedures of both quasi-static method and dynamic method are developed to simulate fracture processes in uniaxial tensile tests conducted on a concrete panel. Cases of different loading rates are compared with the quasi-static case. It is found that the inertia effect due to load increasing becomes less important and can be ignored with the loading rate decreasing, but the inertia effect due to unstable crack propagation remains considerable no matter how low the loading rate is. Therefore, an unrealistic result will be obtained if a fracture process including unstable cracking is simulated by the quasi-static procedure.
\end{abstract}

Keywords Concrete - Generalized beam lattice model . Inertia effects - Quasi-static and dynamic method · Fracture

\section{Introduction}

Analysis of fracture processes in concrete is a timely topic. To conduct numerical investigations, a material model is required firstly to take the material structure into consideration, and then a proper numerical procedure is necessary.

Microstructure in concrete plays a very important role in fracture processes. The characteristic inelastic response of

J. X. Liu $(\varangle) \cdot$ S. C. Deng · N. G. Liang

State Key Laboratory of Nonlinear Mechanics,

Institute of Mechanics, Chinese Academy of Sciences, 100080 Beijing, China

e-mail: liujx@lnm.imech.ac.cn concrete is very difficult to interpret without appealing to their micro-structure (see for instance [9]). Concrete is usually described as a three-phase material, where matrix, aggregate and interface zones are distinguished (see for instance $[11,13,14,17,19,20])$. Among different kinds of microstructural models, lattice type models are being applied by more and more investigators. One important reason is that the lattice model allows a straightforward implementation of the material heterogeneity [13]. Lattice type material modeling has been successfully used for solving classical problems of elasticity [7]. More recently, it has been employed for simulating the progressive failure in heterogeneous media, especially by theoretical physicists $[5,6]$. To investigate fracture processes in concrete, many different types of meso-level lattice models have been developed in the past. As has been generally accepted, it is necessary to project the material structure directly on to the lattice in order to obtain more realistic results $[11,13,20]$. Schorn et al. [18] and Bazant et al. [1] used truss elements, which require some numerical measures to exclude instability during fracture propagation. Schlangen et al. [17] adopted Euler-Bernoulli beams of [6]. Bolander et al. [2] developed a kind of spring element, which is equivalent to the Euler-Bernoulli beam element in a special case. Generally, the Timoshenko beam is more proper than the Euler-Bernoulli beam as each beam in the lattice is short and deep $[11,13]$. However, in order to include the interface zones directly, the mesh size, i.e. the span of beams, must be limited to the same order of magnitude as the interfacial thickness. As a result, this leads to practical difficulties, due to the extremely large computational effort.

In order to solve the problem of computational effort in the beam lattice models, the GB lattice model firstly developed by Liu et al. [14] is adopted. Obviously, according to the basic idea of finite element method, the mesh size has to be of the same order of magnitude as the meso-level characteristic 
length in order to observe the detailed response at this level. This investigation shows that, however, the three-phase material structure can be projected directly on top of a regular triangular GB lattice whose mesh size can be even larger than the maximum aggregate. The key technique is the development of a kind of two-node and three-phase elements, which are different from beam elements, truss elements or springs. Thus, the three-phase element is called generalized beam (GB) element, and the corresponding lattice is called GB lattice. A GB element is composed of three beams. Every beam in GB elements can be a matrix beam, interface beam or aggregate beam. In the beam lattice 10-100 nodes are usually needed to simulate an aggregate, but just a single node is required to model an aggregate in the GB lattice. As a result, computational effort is extremely reduced when the GB lattice model is adopted. The justification of the GB lattice model was done in [14].

Two approaches can be distinguished to deal with fracture of concrete: quasi-static method and dynamic method. In the present investigation, only ideally brittle lattices are discussed. Once a strength criterion is adopted for failure of the elements, a typical procedure for the quasi-static simulation is as follows (see for instance $[6,11,16]$ ). The load is applied gradually and linear elastic analysis is performed until the element with the highest stress-to-strength ratio reaches the prescribed threshold value (see for instance [13]). The critical element is then eliminated and a new analysis is performed after updating the stiffness matrix while the applied load is kept unchanged to check whether another element will fail. If no more elements fail, the calculation is restarted from zero loads again until the complete failure of the specimen. Obviously, the fracture process obtained by the quasi-static method is composed of a series of static equilibrium states. In the dynamic method, critical elements are also eliminated immediately, just as what has been done in the double cantilever beam (DCB) test $[10,15]$. The essential difference between two methods is: fracture processes are simulated by solving the dynamic equations of motion of the system in the dynamic method, but they are obtained by solving the equilibrium equations in the quasi-static method. Additionally, the quasi-static method is much more computationally efficient than the dynamic method because the time step has to be set small enough to guarantee the accuracy of the simulation in the dynamic cases (see for instance [4,21]).

Then, what is the difference between results obtained by the two methods? Are the two kinds of results consistent with each other when the loading rate is enough low? The first question has been investigated by many researchers. For example, Ibrahimbegovic et al. [9] showed that inertial effects have an important role in the crack pattern. The second question is equivalent to the assumption of the quasi-static method. In this paper, cases where the loading rate is respectively $1.0,10^{-1}, 10^{-2}$ and $10^{-3} \mathrm{~m} / \mathrm{s}$ are simulated. Moreover, they are respectively compared with the corresponding quasi-static result. In a dynamic fracture process, there are two kinds of inertia effects: that due to load increasing, and that due to crack propagation. The two inertia effects are discussed in detail. It is found that the inertia effect due to unstable crack propagation still has a considerable influence on fracture processes even though the loading rate is very low. Therefore, the quasi-static method is not suitable to be used to simulate fracture processes including unstable crack propagation.

\section{GB lattice model [14]}

\subsection{The geometry of the model}

At first, the creation of the beam lattice model is recalled. To obtain more realistic results, the material structure is projected directly on to the lattice $[11,20]$. In Fig. 1, if both ends of a beam fall in the matrix (aggregate) phase, it is assigned the equivalent matrix (aggregate) properties. If however one of its ends is in the matrix phase and the other is in the aggregate phase, then it is assigned the equivalent interface properties. The relationship between the properties of a continuum phase and its representative beams in the lattice is obtained by the equivalence of strain energy stored in a unit cell under constant strains (see for instance $[11,16]$ ).

Like the above beam lattice, it is also in a very straightforward way that the GB lattice corresponds to the material structure. In Fig. 2, an aggregate is projected on to a matrix GB lattice, with its center lying on node $i$. The six elements around node $i$ are partly overlain by the aggregate. These parts are assigned the equivalent aggregate properties. The interface is simplified as a thin layer with a rectangular cross-section clinging to the aggregate. The parts of the six elements overlain by the interface are assigned the interfacial properties.

The GB element is apparently a two-node and three-phase element. Every phase of the element is represented by a beam of corresponding (equivalent) properties. For example, in Fig. 2, the six GB elements around node $i$ are all composed of an aggregate beam, an interface beam and a matrix beam. However, it can be imagined that the GB element which is not overlain by any aggregate is actually one-phase, i.e. matrixphase. Nevertheless, in order to make up the deficiency of the relatively coarser GB lattice, the one-phase GB elements are divided into three matrix beams of the same span. When a GB element is overlain by two aggregates, the element is actually composed of two aggregate beams, two interface beams and one matrix beam. For simplification, this kind of elements is considered to be composed of one interface beam and two aggregate beams of the same span. In a word, all GB elements are composed of three beams. 
(a)

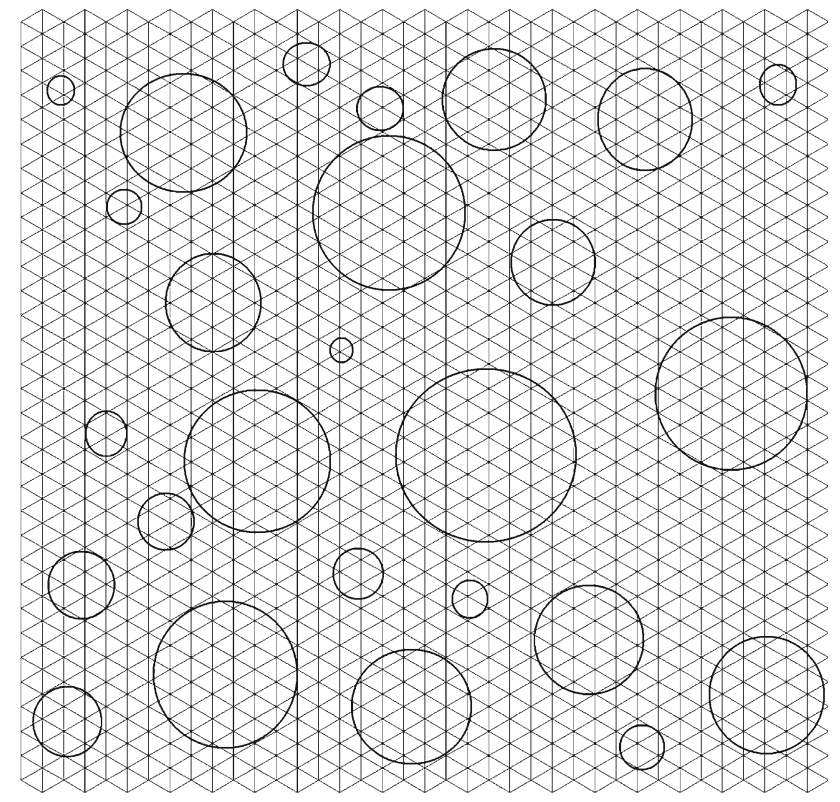

(b)

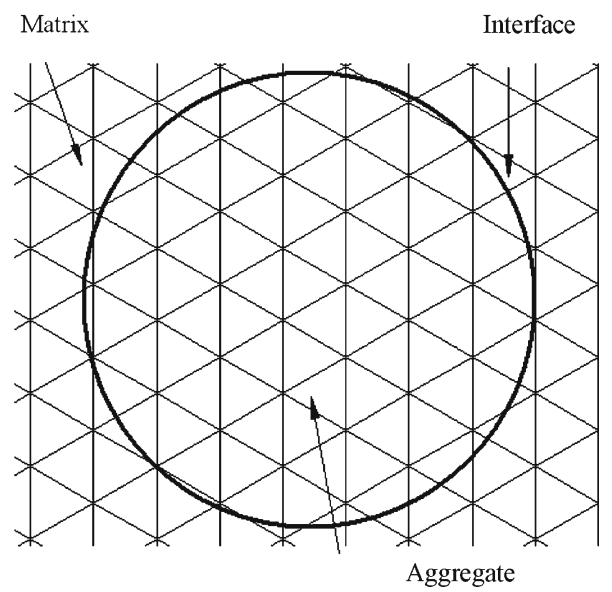

Fig. 1 Particle structure modeled by the beam lattice model: a general particle structure; $\mathbf{b}$ definition of matrix, interface and aggregate element

Found when Fig. 1b is compared with Fig. 2, an aggregate usually covers $10-100$ nodes in the beam lattice, but an aggregate just overlays a single node in the GB lattice. As a result, computational effort is extremely reduced in the GB lattice model.

If three beams in each GB element are assumed to cling firmly to each other and deform together without sliding, the displacements of two ends of the middle beam in the GB element are completely determined by the displacements of the two nodes of the GB element, which will be discussed in detail in Sect. 2.4. Then, the degrees of freedom of two ends of the middle beam are not necessarily included in the discrete system of equations of equilibrium, resulting that computational effort is further reduced.

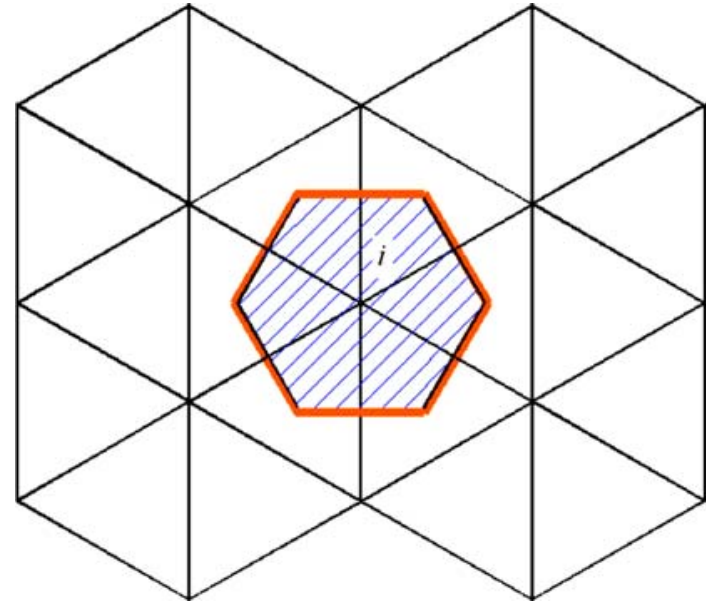

Fig. 2 A GB lattice including particle structure

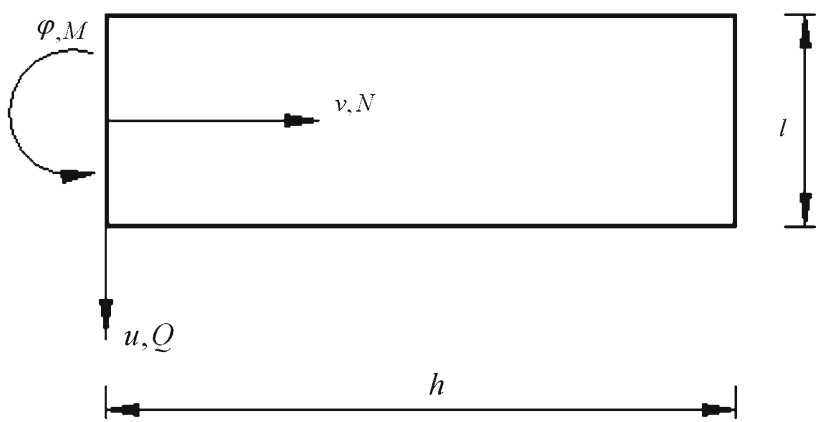

Fig. 3 Kinematics and statics of a beam

\subsection{Stiffness matrices of matrix, aggregate and interface} beams

Matrix beams and aggregate beams are described by Timoshenko beam theory. However, Timoshenko beam theory can not describe interface beams, because the aspect ratio of interface beams is usually too high. In this paper, interface beams are described by Eq. (A1) in Appendix A of [2]. These two kinds of stiffness matrices have the following common expression (Fig. 3):

$\left\{\begin{array}{c}Q_{1} \\ N_{1} \\ M_{1} \\ Q_{2} \\ N_{2} \\ M_{2}\end{array}\right\}=\underbrace{\left[\begin{array}{cccccc}M_{11} & 0 & -M_{34} & -M_{11} & 0 & -M_{34} \\ & M_{22} & 0 & 0 & -M_{22} & 0 \\ & & M_{33} & M_{34} & 0 & M_{36} \\ & & & M_{11} & 0 & M_{34} \\ & & \mathrm{SYM} & & M_{22} & 0 \\ & & & & M_{33}\end{array}\right]}_{M}\left\{\begin{array}{l}u_{1} \\ v_{1} \\ \varphi_{1} \\ u_{2} \\ v_{2} \\ \varphi_{2}\end{array}\right\}$

where, $\mathbf{F}_{12}=\left\{Q_{1} N_{1} M_{1} Q_{2} N_{2} M_{2}\right\}^{T}$ and $\mathbf{u}_{12}=\left\{u_{1} v_{1}\right.$ $\left.\varphi_{1} u_{2} v_{2} \varphi_{2}\right\}^{T}$ are the generalized force vector and the generalized displacement vector, respectively. 
For a Timoshenko beam, the independent elements in Eq. (1) can be expressed in the form $[8,21]$

$M_{11}=\frac{12 E^{(b)} I}{(1+b) h^{3}}, \quad M_{34}=\frac{6 E^{(b)} I}{(1+b) h^{2}}, \quad M_{22}=\frac{E^{(b)} A}{h}$,

$M_{33}=\frac{E^{(b)} I(4+b)}{h(1+b)}, \quad M_{36}=\frac{E^{(b)} I(2-b)}{h(1+b)}$

where, $E^{(b)}$ is the Young's Modulus; $t^{(b)}, h$ and $l$ are respectively the thickness, the span and the height of the Timoshenko beam; $A=t^{(b)} l$ is the cross-section area; $I=t^{(b)} l^{3} / 12$ is the moment of inertia; $b=a E^{(b)} l^{2} / G^{(b)} h^{2}$ is the dimensionless parameter in Timoshenko beam theory; $G^{(b)}=$ $E^{(b)} / 2\left(1+v^{(b)}\right)$ is the modulus of rigidity, where $v^{(b)}$ is the Poisson's ratio.

By comparing Eq. (A1) in Appendix A of [2] with Eq. (1), the independent elements in Eq. (1) can be expressed in the form

$M_{11}=\frac{G^{(b)} A}{h}, \quad M_{34}=\frac{G^{(b)} A}{2}, \quad M_{22}=\frac{E^{(b) \prime} A}{h}$,

$M_{33}=\frac{G^{(b)} A h}{4}+\frac{E^{(b) \prime} I}{h}, \quad M_{36}=\frac{G^{(b)} A h}{4}-\frac{E^{(b) \prime} I}{h}$

where, $E^{(b) \prime}=E^{(b)} /\left[1-\left(v^{(b)}\right)^{2}\right]$.

In the following, a superscript is added to $\mathbf{M}$ in order to declare the material property, i.e. $\mathbf{M}^{\mathbf{m}}, \mathbf{M}^{\mathbf{a}}$ and $\mathbf{M}^{\mathbf{i}}$ are respectively, the stiffness matrix of matrix, aggregate and interface beams. When an aggregate is very small, the interface beams clinging to the aggregate have a relatively lower aspect ratio. In this case, it is more proper to take these interface beams as Timoshenko beams. However, in the present investigation, for simplification, all interface-phase beams adopt the stiffness matrix in Eq. (3).

\subsection{Parameter calibration of the regular triangular GB lattice}

Spans of three beams of each GB element are determined as soon as the particle overlay [20] is projected on top of the GB lattice. To an interface beam, the properties of interface material themselves instead of its equivalent properties are assigned. Moreover, the depth of the interface beam can be calculated in the form

$l= \begin{cases}2 d_{i} / \sqrt{3} & \left(d_{i} \leq L / 2\right) \\ 2\left(L-d_{i}\right) / \sqrt{3} & \left(L / 2 \leq d_{i} \leq L\right)\end{cases}$

where, $L$ is the length of the GB element, and $d_{i}$ denotes the distance between node $i$ and the middle point of the interface beam along its span-direction, in the GB element $i-j$.

Then we introduce how to calculate the geometrical and material properties of both matrix and aggregate beams. The basic idea is based on the equivalence of strain energy stored

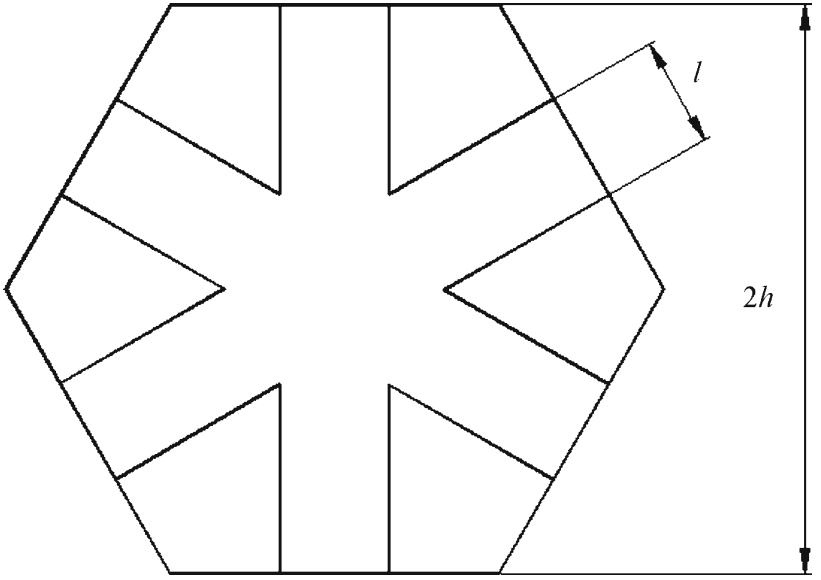

Fig. 4 A triangular matrix/aggregate GB lattice with a hexagonal unit cell

in a unit cell (Fig. 4) of a lattice with its continuum counterpart under constant strains

$U_{\text {cell }}=U_{\text {continuum }}$

The detailed process has been presented by both OstojaStarzewski [16] and Karihaloo et al. [11] to obtain the relationship between the triangular Timoshenko beam lattice and its micropolar continuum equivalent. In the present investigation, the result of Karihaloo et al. [11] is used directly in our parameter system (Fig. 4):

$$
\begin{aligned}
& \frac{E}{E^{(b)}}=\sqrt{3}\left(\frac{l}{h}\right) \frac{t^{(b)}}{t} \frac{4+\left(\frac{l}{h}\right)^{2} \frac{1}{1+b}}{12+\left(\frac{l}{h}\right)^{2} \frac{1}{1+b}}, \\
& v=\frac{4-\left(\frac{l}{h}\right)^{2} \frac{1}{1+b}}{12+\left(\frac{l}{h}\right)^{2} \frac{1}{1+b}}
\end{aligned}
$$

where, $E^{(b)}$ and $E$ are the Young's modulus of the Timoshenko beams and its continuum equivalent. $h$ and $l$ are the span and depth of the Timoshenko beam. $t^{(b)}$ and $t$ are the thickness of the Timoshenko beam and its continuum equivalent. Moreover,

$b=\frac{12+11 v^{(b)}}{20}\left(\frac{l}{h}\right)^{2}$

The second equation of Eq. (6) can be rewritten in the form

$b=\frac{1+v}{4(1-3 v)}\left(\frac{l}{h}\right)^{2}-1$

Substitution of Eq. (7) into the second equation of Eq. (6) yields the result

$v^{(b)}=\frac{5(1+v)}{11(1-3 v)}-\frac{20}{11}\left(\frac{l}{h}\right)^{-2}-\frac{12}{11}$ 


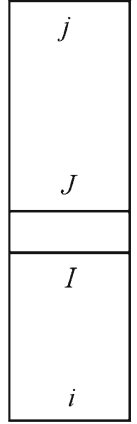

(a)

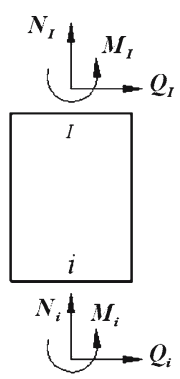

(b)

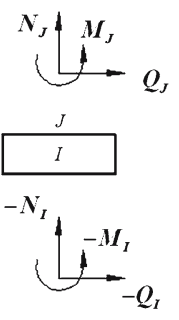

(c)

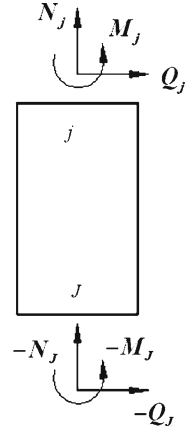

(d)
Fig. 5 A GB element composed of an aggregate beam, an interface beam and a matrix beam: a the GB element; $\mathbf{b}$ the aggregate beam; $\mathbf{c}$ the interface beam; and $\mathbf{d}$ the matrix beam

Substitution of Eq. (8) into the first equation of Eq. (6) yields the result

$E^{(b)}=\frac{2}{\sqrt{3}}\left(\frac{l}{h}\right)^{-1} \frac{t}{t^{(b)}} \frac{E}{1-v}$

In this investigation, all matrix beams and aggregate beams have rectangular cross-sections, the same aspect ratio $l / h=$ 1 , and the same thickness $t^{(b)}=t$. The Poisson's ratio of both matrix and aggregate is $11 / 40$. Then, from Eqs. (9) and (10), their equivalent material properties $v^{(b)}$ and $E^{(b)}$ respectively become

$v^{(b)}=\frac{31}{77}$

$E^{(b)}=\frac{80}{29 \sqrt{3}} E$

\subsection{The stiffness matrix of a GB element}

This section is going to introduce how to determine the stiffness matrix of a GB element from its three beams. For the sake of simplification and without loss of generality, a GB element composed of one aggregate beam, one interface beam and one matrix beam is under investigation (Fig. 5).

In the GB element $i-j$ (Fig. 5a), when external generalized forces are applied on node $i$ and $j$, the relationship between the generalized nodal forces and the generalized nodal displacements can be expressed in the form

$$
\begin{aligned}
\mathbf{F}_{i j} & =\mathbf{K u}_{i j} \\
F_{i j} & =\left\{\begin{array}{llllll}
Q_{i} & N_{i} & M_{i} & Q_{j} & N_{j} & M_{j}
\end{array}\right\}^{T} \\
u_{i j} & =\left\{\begin{array}{llllll}
u_{i} & v_{i} & \varphi_{i} & u_{j} & v_{j} & \varphi_{j}
\end{array}\right\}^{T}
\end{aligned}
$$

where, $\mathbf{K}$, a symmetric matrix, denotes the stiffness matrix of the GB element.

Analogously, for the aggregate beam $i-I$ (Fig. 5b), the interface beam $I-J$ (Fig. 5c), and the matrix beam $J-j$ (Fig. 5d), the relationships between the kinematics and statics can be expressed in the forms, respectively

$i-I$ :

$\mathbf{F}_{i I}=\mathbf{M}^{\mathbf{a}} \mathbf{u}_{i I}$

$\mathbf{F}_{i I}=\left\{\begin{array}{llllll}Q_{i} & N_{i} & M_{i} & Q_{I} & N_{I} & M_{I}\end{array}\right\}^{T}$

$\mathbf{u}_{i I}=\left\{\begin{array}{llllll}u_{i} & v_{i} & \varphi_{i} & u_{I} & v_{I} & \varphi_{I}\end{array}\right\}^{T}$

$I-J$ :

$\mathbf{F}_{I J}=\mathbf{M}^{\mathrm{i}} \mathbf{u}_{I J}$

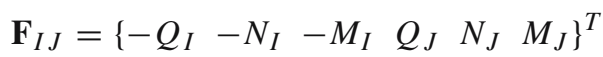

$\mathbf{u}_{I J}=\left\{\begin{array}{llllll}u_{I} & v_{I} & \varphi_{I} & u_{J} & v_{J} & \varphi_{J}\end{array}\right\}^{T}$

$J-j$ :

$\mathbf{F}_{J j}=\mathbf{M}^{m} \mathbf{u}_{J j}$

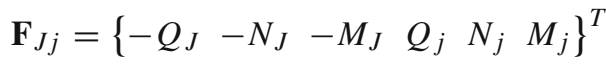

$\mathbf{u}_{J j}=\left\{\begin{array}{llllll}u_{J} & v_{J} & \varphi_{J} & u_{j} & v_{j} & \varphi_{j}\end{array}\right\}^{T}$

In consideration of the equilibrium conditions at $I$ and $J$, a system of algebraic equations expressed in the generalized displacement vector $\mathbf{u}_{I J}$ can be obtained when $\mathbf{u}_{i j}$ are assumed to be known

$$
\underbrace{\left[\begin{array}{cc}
\mathbf{M}_{\mathbf{I I I}}^{\mathbf{a}}+\mathbf{M}_{\mathbf{I}}^{\mathbf{i}} & \mathbf{M}_{\mathbf{I I}}^{\mathbf{i}} \\
\left(\mathbf{M}_{\mathbf{I I}}^{\mathbf{i}}\right)^{T} & \mathbf{M}_{\mathbf{I I I}}^{\mathbf{i}}+\mathbf{M}_{\mathbf{I}}^{\mathbf{m}}
\end{array}\right]}_{\mathbf{A}} \mathbf{u}_{I J}^{\left[\begin{array}{cc}
-\left(\mathbf{M}_{\mathbf{I I}}^{\mathbf{a}}\right)^{T} & 0 \\
0 & -\mathbf{M}_{\mathbf{I I}}^{\mathbf{m}}
\end{array}\right]} \mathbf{u}_{i j}
$$

where, $\mathbf{M}_{\mathbf{I}}^{\mathbf{a}}, \mathbf{M}_{\mathbf{I I}}^{\mathbf{a}}$ and $\mathbf{M}_{\mathrm{III}}^{\mathrm{a}}$ denote the top-left, top-right and bottom-right $3 \times 3$ sub-matrix of $\mathbf{M}^{\mathbf{a}}$, respectively, and the same denotation rule is also used to $\mathbf{M}^{\mathbf{i}}$ and $\mathbf{M}^{\mathbf{m}}$. Both $\mathbf{A}$ and B are $6 \times 6$ matrices.

By solving the system of Eq. (17), the relationship between $\mathbf{u}_{I J}$ and $\mathbf{u}_{i j}$ can be expressed in the form

$\mathbf{u}_{I J}=\underbrace{\mathbf{A}^{-1} \mathbf{B}}_{\mathbf{R}} \mathbf{u}_{i j}$

where, $\mathbf{R}=\mathbf{A}^{-\mathbf{1}} \mathbf{B}$.

$\mathbf{R}_{\mathbf{I}}, \mathbf{R}_{\text {II }}$ and $\mathbf{R}_{\text {III }}$ respectively denote the top-left, top-right, bottom-right $3 \times 3$ sub-matrix of $\mathbf{R}$. After a lengthy but elementary calculation, the stiffness matrix of the GB element $\mathbf{K}$ can be expressed in the form

$\mathbf{K}=\left[\begin{array}{ll}\mathbf{M}_{\mathbf{I}}^{\mathrm{a}}+\mathbf{M}_{\mathrm{II}}^{\mathrm{a}} \mathbf{R}_{\mathbf{I}} & \mathbf{M}_{\mathrm{II}}^{\mathrm{a}} \mathbf{R}_{\mathbf{I I}} \\ \mathbf{S Y M} & \left(\mathbf{M}_{\mathbf{I I}}^{\mathrm{m}}\right)^{T} \mathbf{R}_{\mathbf{I I I}}+\mathbf{M}_{\mathrm{III}}^{\mathrm{m}}\end{array}\right]$

\section{Quasi-static method and dynamic method to simulate fracture processes}

\subsection{Quasi-static method}

As pointed out in the introduction, fracture is simulated by subsequent removal of critical elements from a lattice. Three 
beams in every GB element are judged whether to be critical independently. Once a beam approaches criticality, the corresponding GB element becomes a critical element. A criterion must be set to decide when a beam must be removed. In this paper the maximum tensile stress in each beam is computed in the following form (see for instance [20])

$\sigma_{e f f}=\frac{N}{A}+\alpha \frac{\left(\left|M_{i}\right|,\left|M_{j}\right|\right)_{\max }}{W}<f_{t}$

where, $N$ is the normal force in the considered beam, $M_{i}$ and $M_{j}$ are the bending moments at the nodes $i$ and $j$ of the beam, and $W=t^{(b)} l^{2} / 6$ is the section modulus. The coefficient $\alpha$ regulates what part of the bending moment is considered. In this paper, $\alpha$ is $0.005[11,13,19,20]$. When the effective stress of a particular beam reaches its tensile strength $f_{t}$, brittle fracture is simulated by instantaneously removing the beam from the lattice.

\subsection{Dynamic method}

The dynamic equations of motion of the system can be expressed in the form

$\mathbf{M} \ddot{\mathbf{u}}(t)+\mathbf{C} \dot{\mathbf{u}}(t)+\mathbf{K u}(t)=\mathbf{Q}(t)$

The symbols in Eq. (21) are explained one by one in the following.

(1) $\mathbf{M}$ is the mass matrix of the lattice. It is assumed that mass of each GB element concentrates on its ends in the triangular GB lattice. As a result, $\mathbf{M}$ can be written as

$$
\mathbf{M}=\operatorname{diag}\left(m_{1}, m_{1}, J_{1}, m_{2}, m_{2}, J_{2}, \ldots, m_{n}, m_{n}, J_{n}\right)
$$

where $n$ is the total of nodes. The diagonal matrix $\mathbf{M}$ is $3 n \times 3 n$. For the sake of simplicity, it is assumed that the three phases have the same density $\rho$. Then, $m_{i}$ and $J_{i}$ can be written as

$$
\begin{aligned}
m_{i} & =\frac{\sqrt{3} \rho L^{2} t}{2} \\
J_{i} & =\frac{5 \rho L^{4} t}{8}\left(\frac{\sqrt{3}}{9}-\frac{3 \pi}{64}\right)(i=1,2, \ldots, n)
\end{aligned}
$$

(2) $\mathbf{u}(t)$ is the vector of nodal displacements at time $t$ :

$$
\begin{aligned}
& \mathbf{u}(t)=\left\{\begin{array}{llllll}
u_{1}(t) & v_{1}(t) & \varphi_{1}(t) & u_{2}(t) & v_{2}(t) & \varphi_{2}(t)
\end{array}\right. \\
& \left.\times \ldots u_{n}(t) v_{n}(t) \varphi_{n}(t)\right\}^{T}
\end{aligned}
$$

Analogously, $\dot{\mathbf{u}}(t)$ and $\ddot{\mathbf{u}}(t)$ are the vector of nodal velocities and the vector of nodal accelerations, respectively:

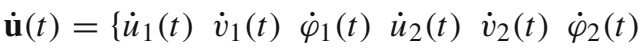

$$
\begin{aligned}
& \left.\times \ldots \dot{u}_{n}(t) \dot{v}_{n}(t) \dot{\varphi}_{n}(t)\right\}^{T}
\end{aligned}
$$

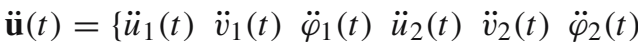

$$
\begin{aligned}
& \left.\times \ldots \ddot{u}_{n}(t) \ddot{v}_{n}(t) \ddot{\varphi}_{n}(t)\right\}^{T}
\end{aligned}
$$

(3) $\mathbf{K}$ is the stiffness matrix of the lattice.

(4) $\mathbf{C}$ is the Rayleigh damping matrix of the lattice and can be written as

$$
\mathbf{C}=\alpha \mathbf{M}+\beta \mathbf{K}
$$

where, $\alpha=0.5 \mathrm{~s}^{-1}$, and $\beta=10^{-4} \mathrm{~ms}$, which are the same as those in [9].

(5) $\mathbf{Q}(t)$ is the total load vector.

In this work, the differential equations of motion in Eq. (21) are solved by using the central difference method. In the method, velocity and acceleration can by expressed as

$\dot{\mathbf{u}}(t)=\frac{1}{2 \Delta t}[\mathbf{u}(t+\Delta t)-\mathbf{u}(t-\Delta t)]$

$\ddot{\mathbf{u}}(t)=\frac{1}{\Delta t^{2}}[\mathbf{u}(t-\Delta t)-2 \mathbf{u}(t)+\mathbf{u}(t+\Delta t)]$

where, $\Delta t$ is the time step.

Substitution of Eqs. (27), (28) and (29) into Eq. (21) yields the result as

$$
\begin{aligned}
& \left(\frac{1}{\Delta t^{2}} \mathbf{M}+\frac{\alpha}{2 \Delta t} \mathbf{M}+\frac{\beta}{2 \Delta t} \mathbf{K}\right) \mathbf{u}(t+\Delta t) \\
& =\mathbf{Q}(t)+\frac{2}{\Delta t^{2}} \mathbf{M u}(t)-\mathbf{K u}(t)-\left(\frac{1}{\Delta t^{2}}-\frac{\alpha}{2 \Delta t}\right) \\
& \quad \times \mathbf{M u}(t-\Delta t)+\frac{\beta}{2 \Delta t} \mathbf{K u}(t-\Delta t)
\end{aligned}
$$

In the dynamic simulations, the strength criterion in Eq. (20) is adopted. When the effective stress of a particular beam reaches its tensile strength $f_{t}$, brittle fracture is simulated by instantaneously removing the beam from the lattice. Then a new time step is solved $[10,15]$.

\section{Numerical examples and discussions}

Uniaxial tensile experiments conducted on a concrete plate were analyzed by van Mier et al. [20] and Karihaloo et al. [11] by using the beam lattice model. The same experiment on a GB lattice developed by Liu et al. [14] will be simulated by the quasi-static method and dynamic method, respectively. 
Table 1 Elastic properties of phases

\begin{tabular}{lcc}
\hline & $E(\mathrm{MPa})$ & $f_{t}(\mathrm{MPa})$ \\
\hline Aggregate & 70,000 & 10.0 \\
Interface & 25,000 & 1.25 \\
Matrix & 25,000 & 5.0 \\
\hline
\end{tabular}

The difference between two kinds of results is discussed in detail.

The plate is $210 \sqrt{3} \times 210 \sqrt{3} \mathrm{~cm}^{2}$. A regular triangular GB lattice having a total of $1,593 \mathrm{~GB}$ elements and 562 nodes is used in simulation. All elements are $10 \sqrt{3} \mathrm{~cm}$ long. The span of all interface beams is $\sqrt{3} \mathrm{~cm}$. one hundred and twelve particles with diameter $10 \sqrt{3} \mathrm{~cm}$ are projected randomly on to the GB lattice. The time step $\Delta t$ is $=10^{-6} \mathrm{~s}$. In fact, the choice of the time step depends on the characteristic motion time of the smallest element (see for instance [4,21]). Therefore, to avoid a too small time step, the adopted lattice is very coarse and the sizes of both particles and interface are much bigger than realistic concretes. Nevertheless, such a numerical experiment is useful for a qualitative understanding of dynamic fracture processes. Material properties are shown in Table 1. Three phases have the common density $\rho=10^{3} \mathrm{~kg} / \mathrm{m}^{3}$. All translational degrees of freedom are fixed along the left edge, while a uniaxial tension is applied to the right edge through a controlled displacement.

Five cases are analyzed. Case 1 is simulated by the quasistatic method. The other four cases, i.e. case $2-5$ with the loading rate being $1.0,10^{-1}, 10^{-2}$ and $10^{-3} \mathrm{~m} / \mathrm{s}$, respectively, are done by the dynamic method.

\subsection{Results}

Figure 6 shows the $P-\delta$ curve and crack patterns at typical load levels in case 1. The inset of Fig. $6 a$ is the enlarged image from point a to $\mathrm{p}$. Figures 7, 8, 9, and 10 show the comparison between the $P-\delta$ curves of the four dynamic processes and the quasi-static curve, and also the crack patterns at typical load levels.

\subsection{Effect of inertia effects on the fracture processes}

The five cases are analyzed one by one in the following.

\section{(1) Case 1.}

At the peak load level (point a in Fig. 6a), localized crack appears near the middle of the specimen due to failure of interface beams (Fig. 6b). Then, a series of steep drops in load follow even though the increase of the controlled displacement is very small. This kind of steep drops corresponds to unstable crack propaga- tions. In Fig. 6c, the damaged band has formed throughout the specimen. With the controlled displacement further increasing, the damaged band becomes a macro crack (Fig. 6d). In Fig. 6e, the crack nearly separates the specimen into two parts. For the quasi-static case, the main crack progresses through by connecting the jaggered profile of the successive weakest links [9]. In other words, the crack path is the weakest path in the specimen.

(2) Case 2.

In the beginning of the curve, the slope of the elastic regime is much larger than that in case 1 (Fig. 7a), which is also observed in dynamic physical experiments $[3,12]$. The peak load is nearly four times bigger than that in case 1 . Moreover, at the peak load level, the controlled displacement is $6.90 \times 10^{-5} \mathrm{~m}$, which is much smaller than $2.77 \times 10^{-4} \mathrm{~m}$ in case 1 .

The above feathers can be explained as follows. The loading rate, $1.0 \mathrm{~m} / \mathrm{s}$, is so high that the deformation has not enough time to distribute throughout the specimen. Therefore, deformation concentration appears near the displacement-controlled edge, leading to the failure of GB elements near the edge (Fig. 7b, c). On the other hand, this kind of failures makes the propagation of deformation from the controlled edge into the specimen more difficult. In the quasi-static case, the crack path depends only on three-phase material structure. In the present case with a high loading rate, however, crack pattern depends on both the material structure and the inertia effect due to load increasing, and the latter plays a more important role than the former. Elements fail near the controlled edge (Fig. 7b). The local crack appears in the pure matrix region (Fig. 7c). The crack path is smoother than that in case 1 because the inertial effects appear to even out the material structure (Fig. 7d) [9]. Figure 7e shows the crack pattern at the load level d.

(3) Case 3.

In Fig. 8a, both the elastic slope and the peak load are still larger than those of case 1 . However, the differences have reduced obviously when compared with the differences between case 2 and case 1 in Fig. 7a. $P-\delta$ curves of case 3 and case 1 have the approximately similar up-down tendency except that the curve of case 3 has a strong vibration throughout the whole experimental process (Fig. 8a). Now, material structure plays a dominant role in the crack path instead of deformation concentration due to load increasing as in case 2 . Therefore, in Fig. 8b and c, the path of the localized crack is nearly consistent with that in the quasi-static case 1 . However, case 3 has different failure details along the path, which can be found when comparing Fig. 8d with Fig. 6e. When the crack runs through the specimen, a 
Fig. 6 In case 1 , a the $P-\delta$ curve; and crack patterns at four load levels: b point $a$; $\mathbf{c}$ point $b$; d point $\mathrm{c}$; and e point $\mathrm{d}$
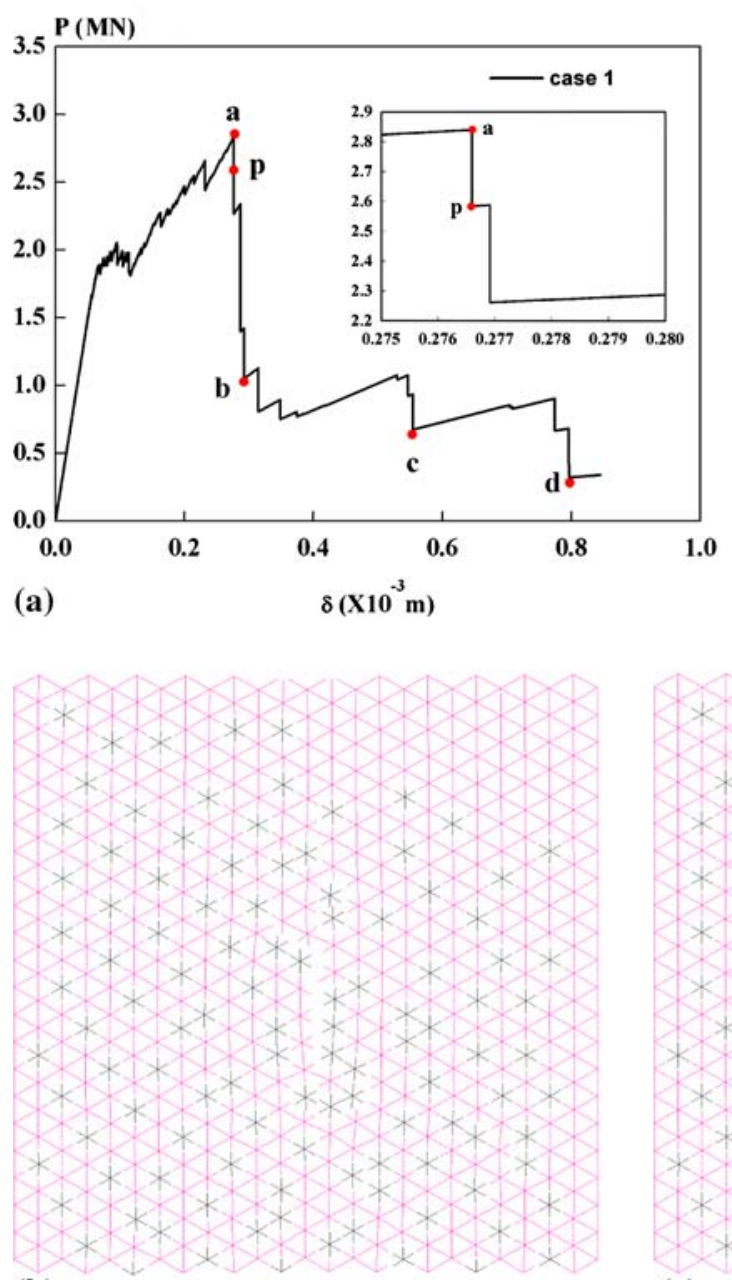

(b)

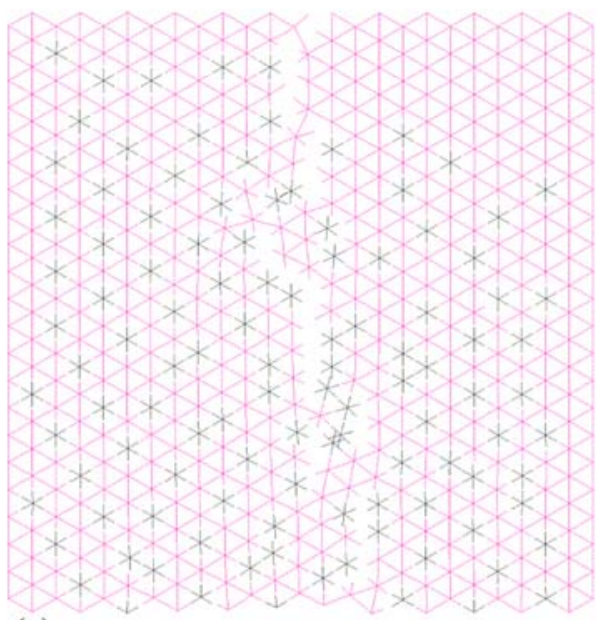

(c)

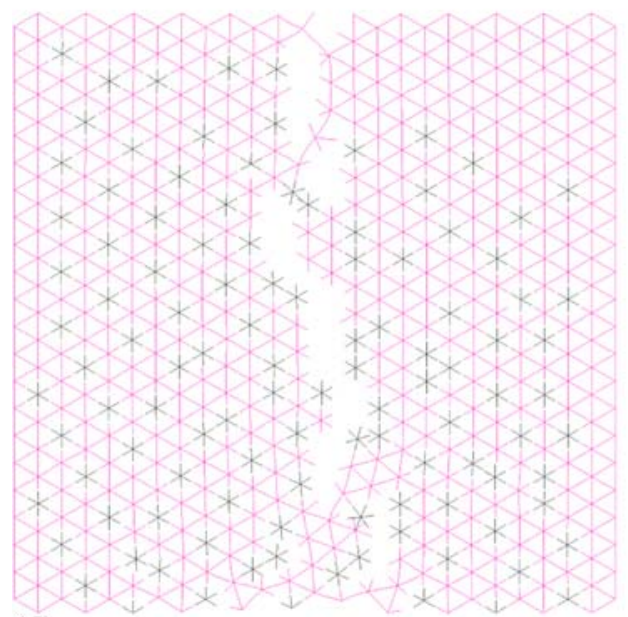

(d)

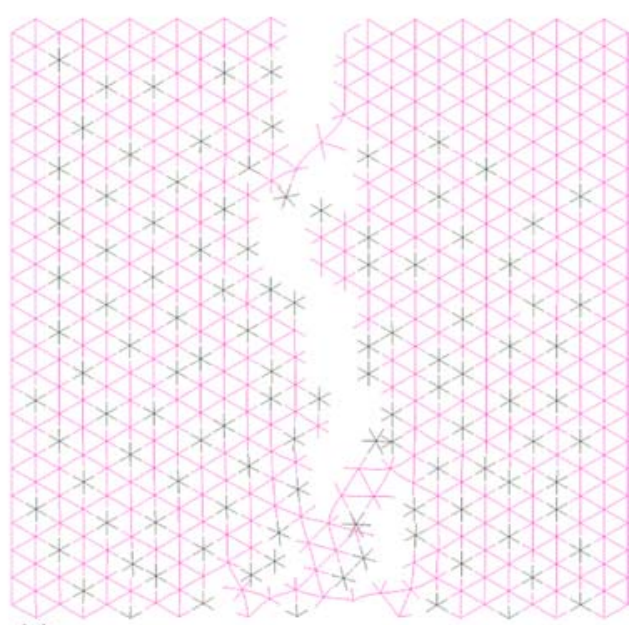

(e)

the edge, load $\mathrm{P}$ will be reduced. In the neighborhood of point $\mathrm{c}$ in Fig. 8a, the compression wave even leads to a negative value of load $\mathrm{P}$. compression wave $[3,4,12]$ begins propagating from the newly-produced crack surface to the displacementcontrolled edges. When the compression wave reaches 
Fig. 7 In case 2, a the $P-\delta$ curve compared with the quasi-static curve; and crack patterns at four load levels: b point $\mathrm{a} ; \mathbf{c}$ point $\mathrm{b}$; $\mathbf{d}$ point $\mathrm{c}$; and e point $d$

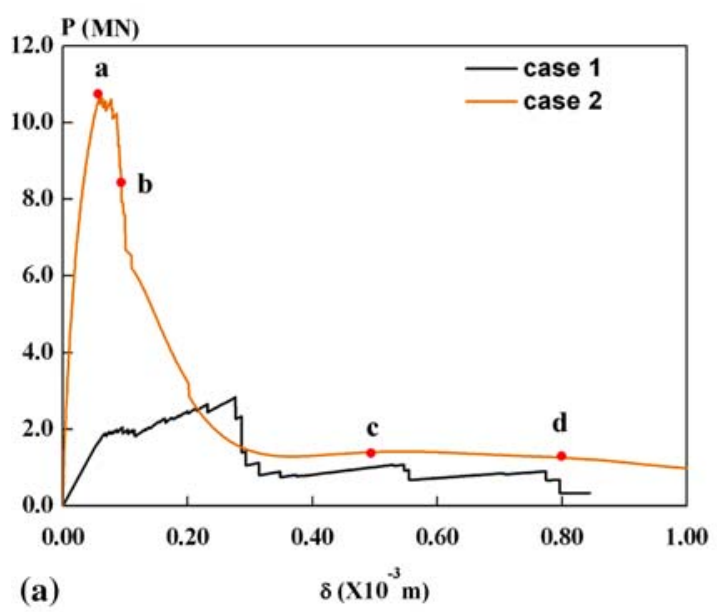

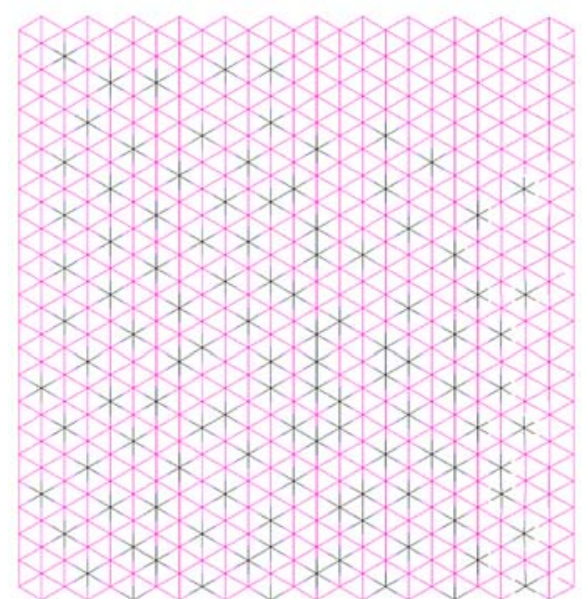

(b)

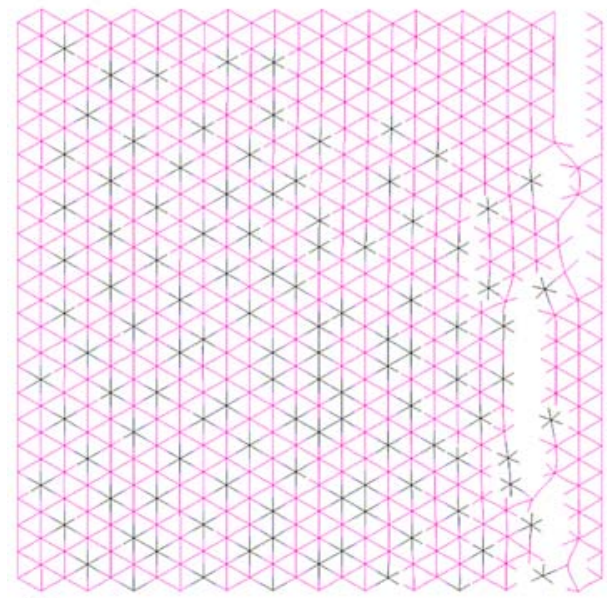

(d)

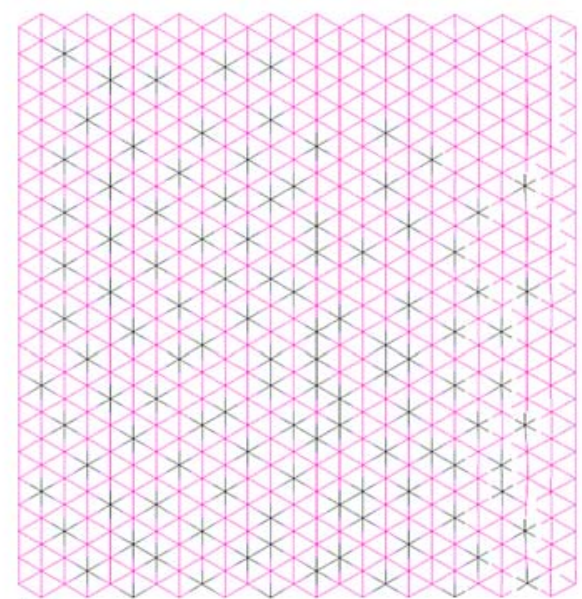

(c)

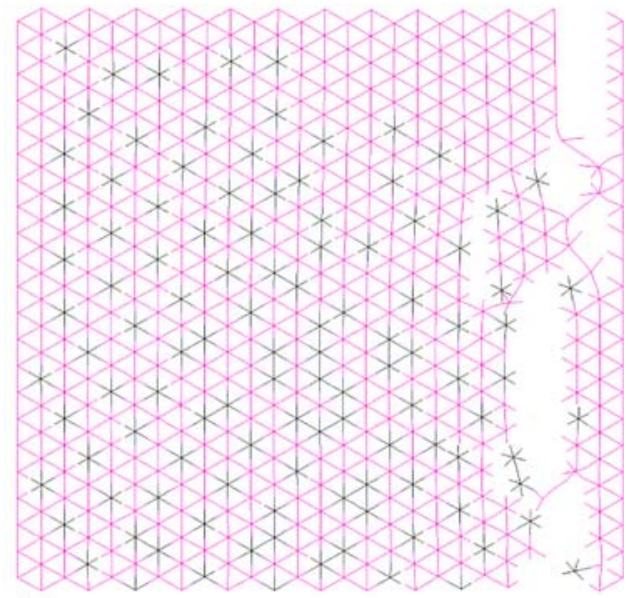

(e)
(4) Case 4.

This case has an elastic slope agreeing to that in case 1 (Fig. 9a). Moreover, case 4 and case 1 have nearly equivalent peak load values: 2.62 and $2.70 \mathrm{MN}$, respectively. However, an obvious difference can be found in the long tail after the peak, i.e. the post-peak regime.
A load vibration directly follows every steep drop, e.g. at point $b$ and $c$ in Fig. 9a. From point a to point $b$, the increase of the controlled-displacement is just $2.84 \times$ $10^{-5} \mathrm{~m}$, and the duration is just $2.84 \times 10^{-3} \mathrm{~s}$. The local crack forms at point a (Fig. 9b), but during the duration $2.84 \times 10^{-3} \mathrm{~s}$ a crack-like band has almost run through 
Fig. 8 In case 3 , a the $P-\delta$ curve compared with the quasi-static curve; and crack patterns at three load levels: b point $a$; c point $b$; and d point $c$

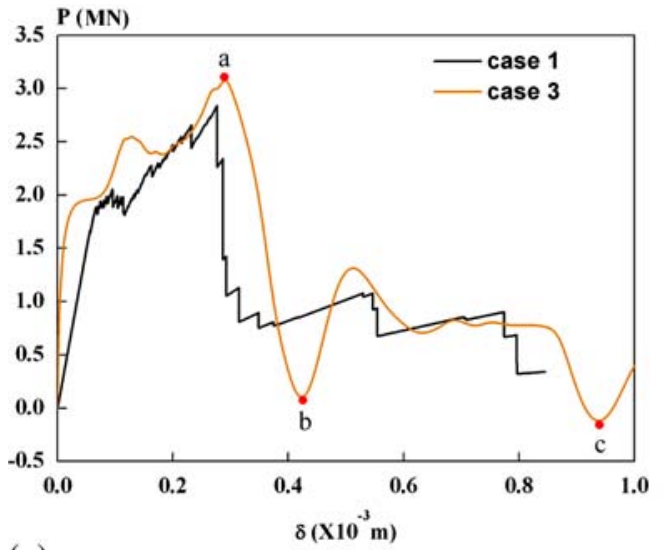

(a)

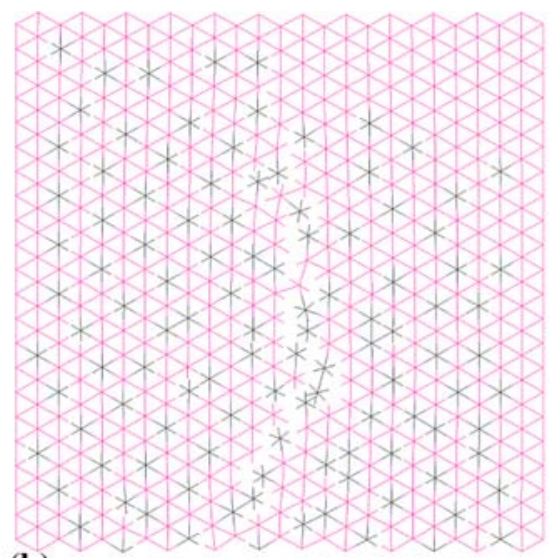

(b)

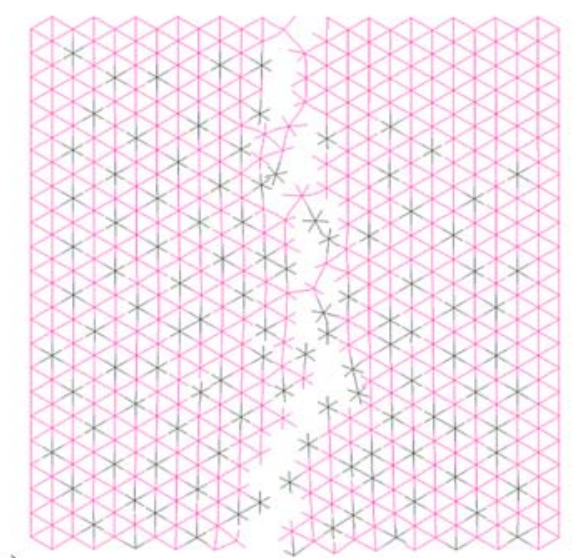

(c)

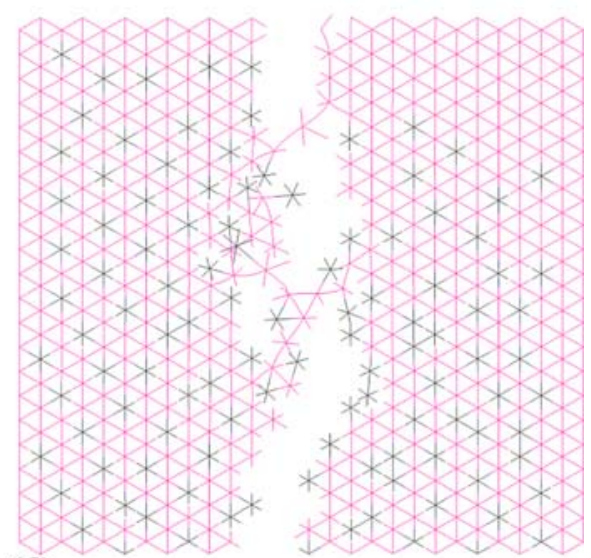

(d) the whole specimen at point b (Fig. 9c). It suggests that the local crack propagates unstably and quickly. Inertia effect due to the unstable propagation leads to the load vibration directly following every steep drop. In the long tail regime, case 4 (Fig. $9 \mathrm{~d}$ and e) and case 1 (Fig. 6d and e) have very different failure details along roughly the same crack path.

Found from Fig. 9a, the load vibration period is roughly $2.5 \times 10^{-3}$ s. In case 2 (Fig. 7), the total experiment time is $10^{-3} \mathrm{~s}$, which is smaller than the vibration period. Therefore, although the loading rate in case 2 is the highest, load vibration due to unstable propagation can't yet be found in Fig. 7a. In case 3 (Fig. 8), the total experiment time is $10^{-2} \mathrm{~s}$, which is equal to several vibration periods, leading to an obvious vibration feather throughout its $P-\delta$ curve. In case 4 , the total experiment time is $10^{-1} \mathrm{~s}$, which is much larger than the vibration period, $2.5 \times 10^{-3} \mathrm{~s}$. As a result, in Fig. 9a, between two steep drops, e.g. between point $b$ and point $\mathrm{c}$, the load vibration takes place near point $\mathrm{b}$, then a roughly line-segment appears until point $\mathrm{c}$, which is very like the zigzag feather of the quasi-static curve.
(5) Case 5 .

In Fig. 10 a, the two $P-\delta$ curves have a good agreement. Moreover, the curve of case 5 also has the zigzag feather similar to the quasi-static curve. However, it is still hard to say that case 5 can be also simulated correctly by the quasi-static method. Actually, in case 5, the inertia effect due to unstable crack propagation still has a considerable influence. From the inset of Fig. 10a, an obvious load vibration occurs after the steep drop near point e, just as discussed in case 4 . This kind of vibration leads to a deviation from the corresponding equilibrium field. Because of the same reason, the crack pattern can be changed. Figure 10b-e shows a cracking process obviously different from that in the quasi-static case (Fig. 6b-e).

4.3 Difference of the two methods in simulating unstable crack propagation

When the loading rate is very low, the inertia effect due to load increasing becomes ignorable, but the inertia effect due to unstable crack propagation remains considerable. During 
Fig. 9 In case 4 , a the $P-\delta$ curve compared with the quasi-static curve; and crack patterns at four load levels: b point $a$; $\mathbf{c}$ point $b ; \mathbf{d}$ point $c$; and e point $d$

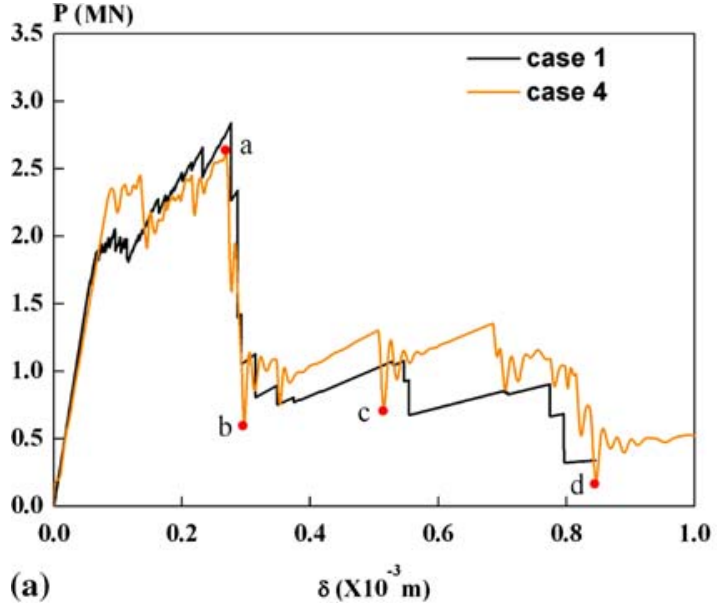

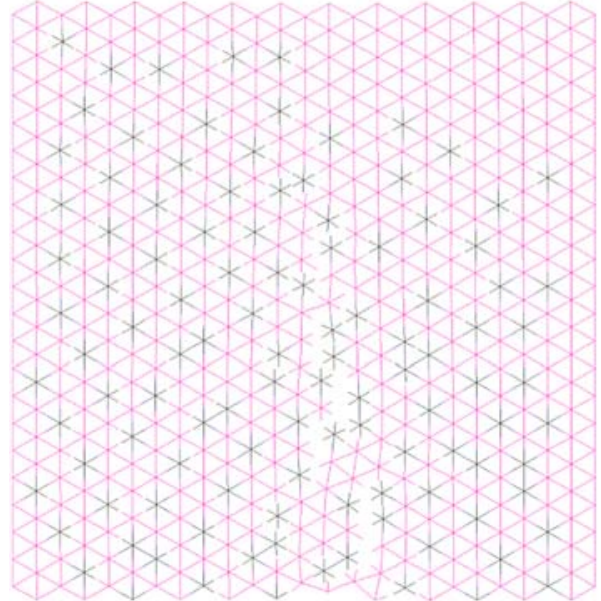

(b)

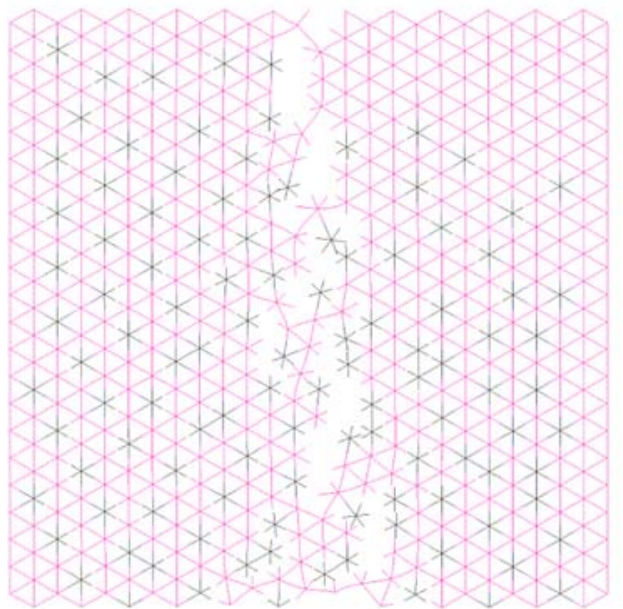

(d)

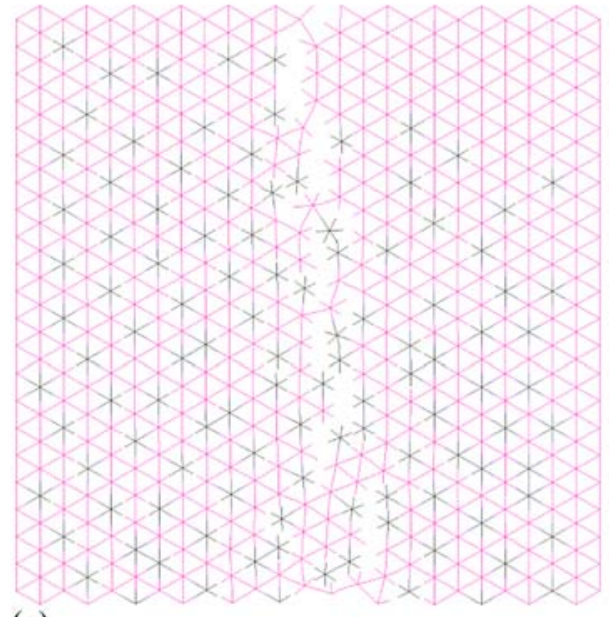

(c)

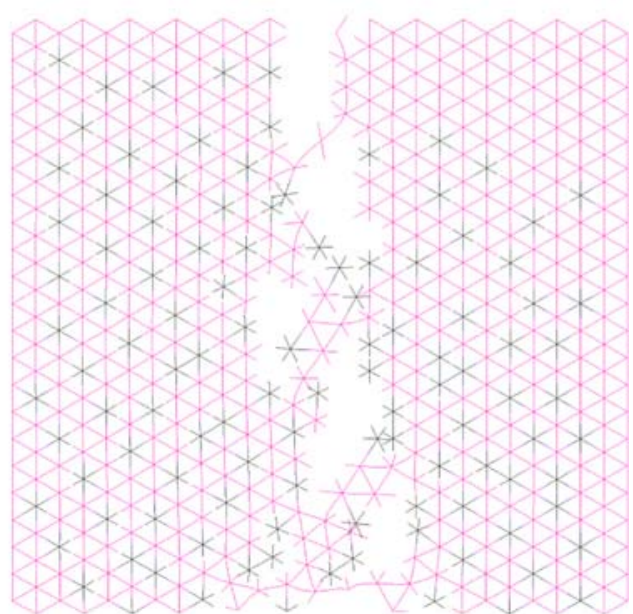

(e) the steep drops, the controlled displacement keeps unchanged in the quasi-static case, but keeps increasing at the loading rate in the four dynamic cases. Although the increase of the controlled displacement is very small due to the steep drops being quick, it is hard to say that the difference between quasi- static and dynamic results is not caused by this increase of the controlled displacement.

To clarify the above question, the following experiment is conducted. The state at point a in Fig. 6a is chosen as the initial state. The dynamic method is employed to simulate 
Fig. 10 In case 5 , a the $P-\delta$ curve compared with the quasi-static curve; and crack patterns at five load levels: b point $\mathrm{a} ; \mathbf{c}$ point $\mathrm{b} ; \mathbf{d}$ point $\mathrm{c}$; e point $\mathrm{d}$; and $\mathbf{f}$ point $\mathrm{e}$

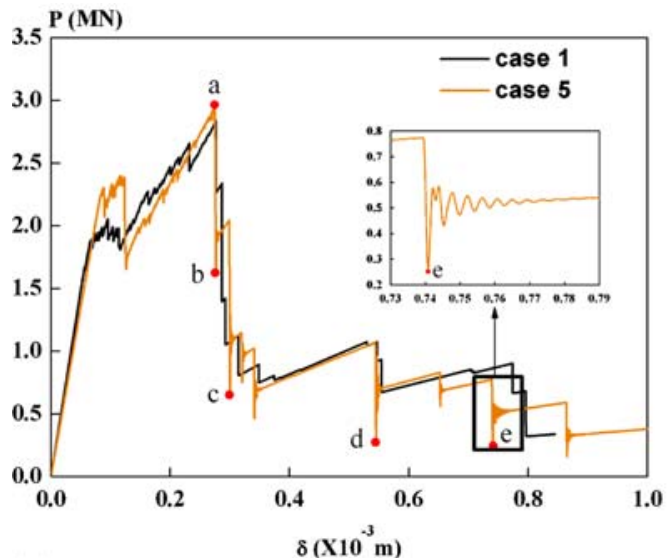

(a)

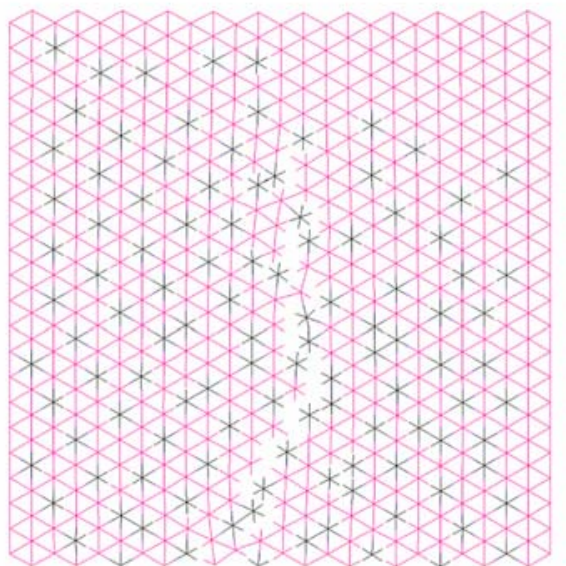

(c)

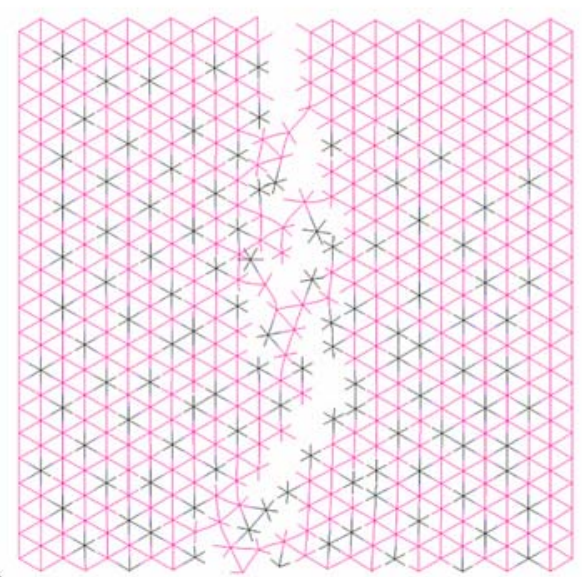

(e)

\section{(d)}

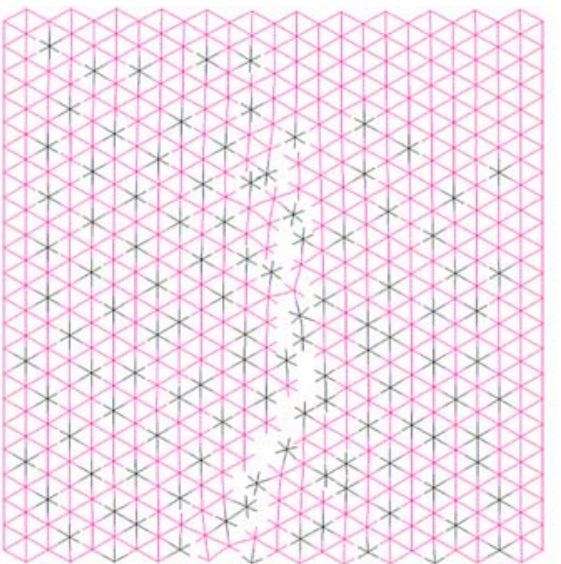

(b)
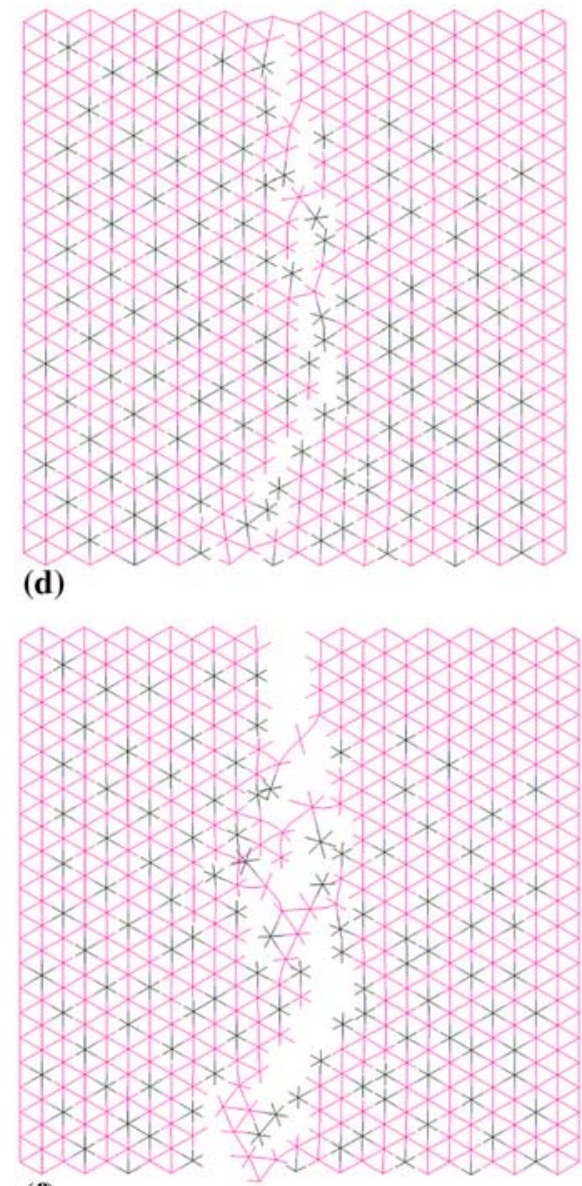

(f) the process directly following point a until reaching a new equilibrium state. The controlled displacement keeps unchanged, i.e. the value at point a, $2.77 \times 10^{-4} \mathrm{~m}$.

The load P is shown versus the time in Fig. 11. In the beginning, the load reduces sharply due to the appearance of failures in succession. In fact, 20 elements fail successively in the whole process. All the failures happen during the period of $0-750 \mu \mathrm{s}$. Then, the load vibration regime follows, and trends to equilibrium under the action of the damping. After
$0.02 \mathrm{~s}$, the load becomes nearly unchanged with time, and the unchanged value is $2.27 \mathrm{MN}$. It indicates that the system has reached a new static equilibrium. In the quasi-static simulation (Fig. 6a), the load of the new equilibrium state denoted by point $\mathrm{p}$ is $2.58 \mathrm{MN}$. Obviously, final equilibrium loads obtained by the quasi-static method and the dynamic method are very different.

Figure $12 \mathrm{a}$ and $\mathrm{b}$ shows the failure sequences of the quasistatic process and the dynamic process respectively, where 


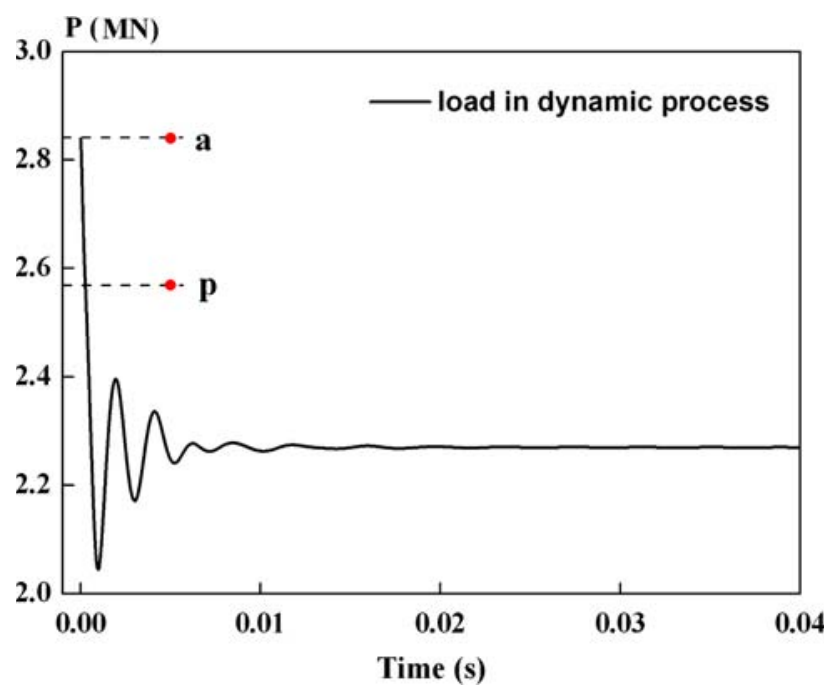

Fig. 11 The load $\mathrm{P}$ versus time during the unstable crack propagation the element denoted by " $\mathrm{A}$ " is the first failed element. In the quasi-static process, failed elements concentrate on a small region (Fig. 12a), and the total of failures is 4 . In the dynamic process, however, failures are almost scattered over the whole specimen, and the total of failures, 20, is much larger. Figure 12c and d shows the crack patterns respectively at the load level p (Fig. 6a) in the quasi-static process and at time $=0.03 \mathrm{~s}$ in the dynamic process. Again, an obvious difference can be found.

In a word, results by the two methods are different even though exactly the same initial state is chosen.

\section{Conclusions}

To solve the problem of computational effort, the generalized beam lattice model was adopted. To simulate fracture processes, both the quasi-static method and dynamic method were introduced, and implemented in a finite element code.
Fig. 12 Comparison of results in the final equilibrium state by the quasi-static method and the dynamic method: distribution and sequence of failures: a the quasi-static method; $\mathbf{b}$ the dynamic method; and crack patterns: c point $p$ in Fig. 6a; and $\mathbf{d}$ at time $=0.03 \mathrm{~s}$ in the dynamic simulation

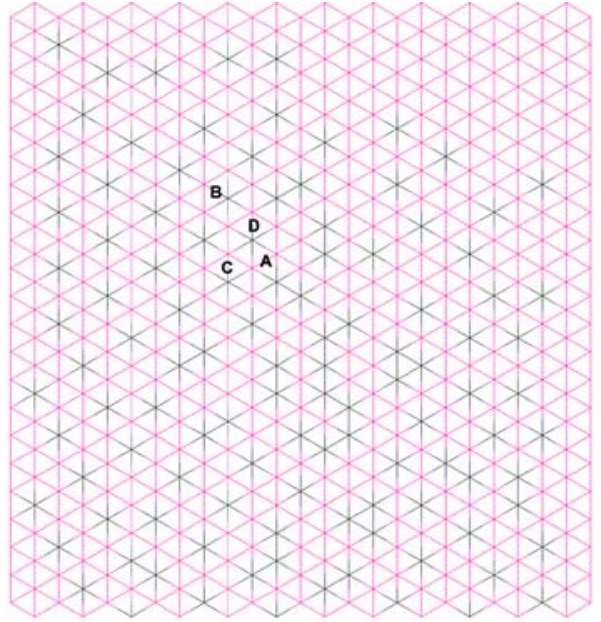

(a)

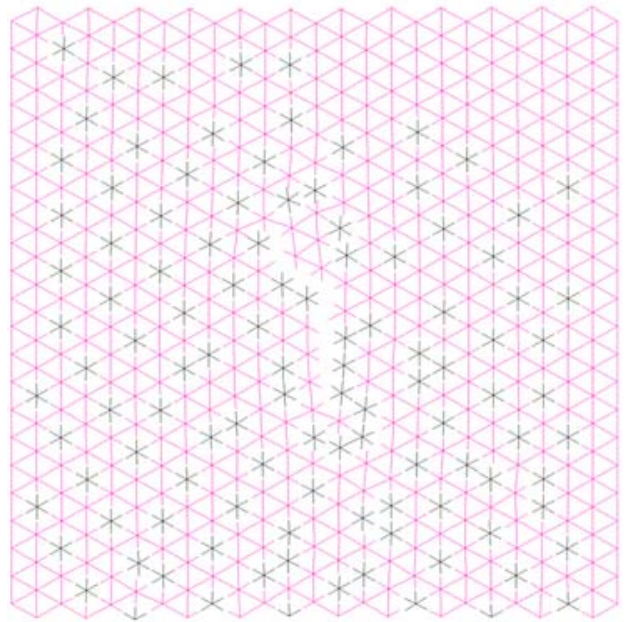

(c)

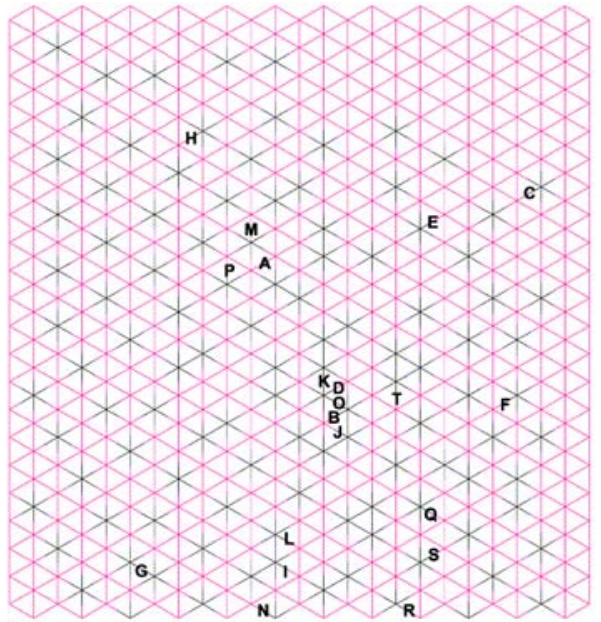

(b)

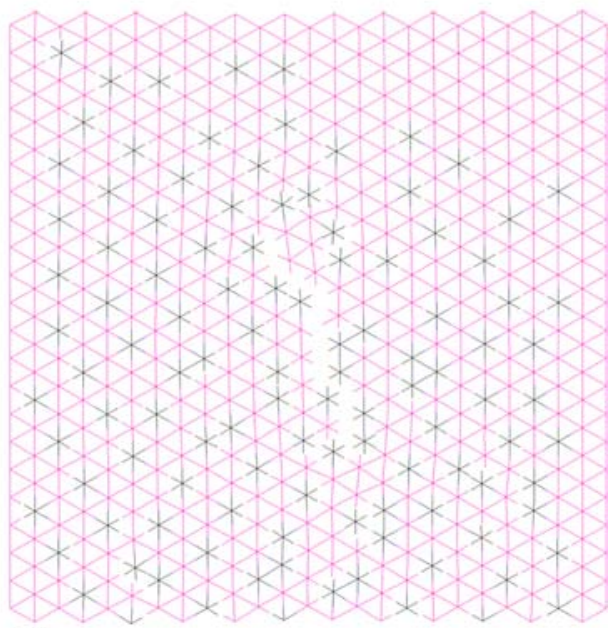

(d) 
Numerical experiments were conducted on a concrete plate subjected to uniaxial tension. Case 1 was simulated by using the quasi-static method. Case $2-5$ with the loading rate being $1.0,10^{-1}, 10^{-2}$ and $10^{-3} \mathrm{~m} / \mathrm{s}$, respectively, were simulated by the dynamic method. The numerical results show that the inertia effect due to load increasing becomes weaker and weaker with the loading rate decreasing. However, the inertia effect due to unstable crack propagation remains considerable in spite of the very low loading rate. Numerical experiment in Sect. 4.3 shows that the quasi-static method can't obtain the same result as the dynamic method in simulating unstable crack propagation.

In a word, it may be questionable that all inertia effects are ignored in the quasi-static method. The inertia effect due to unstable crack propagation, which is still considerable during quasi-static loading processes, is the source of the question. However, simulating a fracture process by the dynamic procedure, requires a substantial increase of computational effort. Therefore, we are developing a new kind of numerical algorithm, combining the quasi-static method and the dynamic method. Results of the new method will be reported in our further investigation.

Acknowledgments This research work was sponsored by National Natural Science Foundation of China through Grant No. 10572140 and No. 10232050, and also partly by Ministry of Science and Technology Foundation No. 2002CB412706. The first author, Jinxing Liu, is grateful to Dr. Xiaojiang Shang, Dr. Nan Liu, Dr. Xiaoliang Chen, Dr. Yinchun Wang for their valuable discussions and suggestions.

\section{References}

1. Bazant ZP, Tabbara MR, Kazemi MT, Pijaudier-Cabot G (1990) Random particle model for fracture of aggregate for fiber composites. ASCE J Eng Mech 116:1686-1705

2. Bolander JE, Saito S (1998) Fracture analyses using spring networks with random geometry. Eng Fract Mech 61:569-591

3. Chu GM, Hu MZ (1983) Stress wave in solids. Intensity and environment newsroom (The collated lectures given by H. Kolsky on visiting China in 1983)
4. Gao HJ, Klein P (1998) Numerical simulation of crack growth in an isotropic solid with randomized internal cohesive bonds. J Mater Phys 46:187-218

5. Herrmann HJ (1991) Patterns and scaling in fracture. In: van Mier JGM, Rots JG, Bakker A (eds) Fracture processes in concrete, rock and ceramics. E\&FN SPON, vol 1, pp 195-211

6. Herrmann HJ, Roux S (1992) Statistical models for the fracture of disordered media. Elsevier, Amsterdam

7. Hrennikoff A (1941) Solution of problems of elasticity by the framework method. J Appl Mech 12:169-175

8. Hughes TJR, Taylor RL, Kanoknukulchai S (1977) A simple and efficient finite element for bending. Int J Numer Eng 11:1529-1543

9. Ibrahimbegovic A, Delaplace A (2003) Microscale and mesoscale discrete models for dynamic fracture of structures built of brittle material. Comput Struct 81:1255-1265

10. Kanninen MF (1974) A dynamic analysis of unstable crack propagation and arrest in the DCB test specimen. Int J Fract 10:415-430

11. Karihaloo BL, Shao PF, Xiao QZ (2003) Lattice modelling of the failure of particle composites. Eng Fract Mech 70:2385-2406

12. Kolsky H (1963) Stress waves in solids. Dover Publications, New York

13. Lilliu G, van Mier JGM (2003) 3D lattice type fracture model for concrete. Eng Fract Mech 70:927-941

14. Liu JX, Deng SC, Zhang J, Liang NG (2007) Lattice-type of fracture model for concrete. Theoretical and applied fracture mechanics (accepted)

15. Malluck JF, King WW (1977) Simulation of fast fracture in the DCB specimen using Kanninen's model. Int J Fract 13:655-665

16. Ostoja-Starzewski M (2002) Lattice models in micromechanics. Appl Mech Rev 55:35-60

17. Schlangen E, van Mier JGM (1992) Experimental and numerical analysis of micromechanisms of fracture of cement-based composites. Cem Concr Compos 14:105-118

18. Schorn H, Rode U (1987) 3-D modeling of process zone in concrete by numerical simulation. In: Shah SP, Swartz SE (eds) Fracture of concrete and rock. Spring, New York pp 220-228

19. van Mier JGM, Chiaia BM, Vervuurt A (1997) Numerical simulation of chaotic and self-organizing damage in brittle disordered materials. Comput Meth Appl Mech Eng 142:189-201

20. van Mier JGM, van Vliet MRA, Wang TK (2002) Fracture mechanisms in particle composites: statistical aspects in lattice type analysis. Mech Mater 34:705-724

21. Wang XC (2003) Finite element method, 1st edn. Tsinghua University Press, Beijing 